\title{
Evaluation of the Esthetic and Functional Outcomes of Extracorporeal Septoplasty for Rhinoplasty in Asian Patients
}

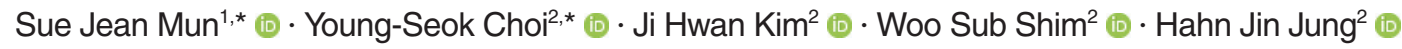 \\ ${ }^{1}$ Department of Otorhinolaryngology-Head and Neck Surgery, Pusan National University Yangsan Hospital, Yangsan; \\ ${ }^{2}$ Department of Otorhinolaryngology-Head and Neck Surgery, Chungbuk National University Hospital,
}

Chungbuk National University College of Medicine, Cheongiu, Korea

\begin{abstract}
Objectives. In extracorporeal septoplasty (ECS), the entire septal cartilage is harvested, and a neo-L strut is built extracorporeally. Thus, ECS can simultaneously achieve septum straightening and tip projection. This study evaluated the functional and esthetic outcomes of the ECS technique for rhinoplasty in Asian patients.

Methods. We conducted a retrospective review of 64 patients who underwent rhinoplasty using ECS between January 2016 and March 2018. Subjective patient satisfaction was assessed and objective surgical outcomes were evaluated. Improvement in nasal obstruction was rated using a visual analog scale (VAS). Anthropometric changes were compared between preoperative and postoperative facial photographs. Complications and revisions were also analyzed.

Results. The participants comprised 64 patients (48 males and 16 females, with a mean age of 29.3 years). The mean operative time was 89.3 minutes. In total, 61 patients were satisfied with the esthetic outcome, and the overall objective rhinoplasty outcome score was 3.45. Preoperative nasal obstruction symptoms (7.9 \pm 1.2 on VAS) improved postoperatively $(3.1 \pm 1.3, P<0.001)$, and significant improvements were also observed in the nasofrontal angle $\left(152.3^{\circ}\right.$ to $\left.148.1^{\circ}, P<0.001\right)$, nasolabial angle $\left(88.8^{\circ}\right.$ to $\left.92.0^{\circ}, P<0.001\right)$, and nasal tip projection $(0.62$ to $0.66, P<0.033)$. Recurrent deviation of the nasal septum and external nose or tip deprojection did not occur in any patients, and there were no cases of revision during the mean follow-up period of 13.3 months.

Conclusion. ECS might be an esthetically and functionally satisfactory alternative rhinoplasty technique for Asian patients with a shortage of septal cartilage.
\end{abstract}

Keywords. Extracorporeal Septoplasty; Rhinoplasty; Asian; Septal Extension Graft

\section{INTRODUCTION}

Tip surgery followed by dorsal augmentation is the most common rhinoplasty procedure for Asian patients because the tip

\footnotetext{
- Received December 4, 2019

Revised April 14, 2020

Accepted April 16, 2020

- Corresponding author: Hahn Jin Jung

Department of Otorhinolaryngology-Head and Neck Surgery, Chungbuk National University Hospital, Chungbuk National University College of

Medicine, 7761 Sunhwan-ro, Heungdeok-gu, Cheongju 28644, Korea

Tel: +82-43-550-3933, Fax: +82-43-556-1090

E-mail: hahnjin2@naver.com

*These authors contributed equally to the study.
}

and the dorsum of the nose are typically low in Asian patients $[1,2]$. Since the noses of Asian patients typically have ethnically specific characteristics, such as insufficient and weak septal cartilage and thick skin, increasing tip projection can be challenging [3-7]. Under these circumstances, septal extension grafts (SEGs) are widely used as an effective procedure for increasing tip projection [8]. However, the use of SEGs in Asian patients has limitations in terms of harvestable cartilage, the possibility of recurrent deviation of the tip in the long term, and thickening of the caudal septum [8].

To address these problems in tip surgery in rhinoplasty in Asian patients, we applied the modified extracorporeal septoplasty (ECS) technique, which is differentiated from the classic

Copyright @ 2021 by Korean Society of Otorhinolaryngology-Head and Neck Surgery.

This is an open-access article distributed under the terms of the Creative Commons Attribution Non-Commercial License (https://creativecommons.org/licenses/by-nc/4.0)

which permits unrestricted non-commercial use, distribution, and reproduction in any medium, provided the original work is properly cited. 
ECS technique by leaving the remaining few millimeters of the dorsal stump in situ to avoid injury to the keystone [9]. In the modified ECS technique, the septum can be harvested to the maximum extent, and a straight neo-L strut is built extracorporeally. The strut provides strong septal support to prevent recurrent deviation and tip projection without thickening of the caudal septum. This study aimed to present our experiences with the modified ECS technique for rhinoplasty in Asian patients and to assess the esthetic outcomes, including tip projection, using both subjective and objective measurements. The functional outcomes of modified ECS were also evaluated.

\section{MATERIALS AND METHODS}

\section{Patients and study design}

This study included 64 patients (48 males and 16 females) who underwent rhinoplasty using the ECS technique between January 2016 and March 2018. We retrospectively reviewed their medical records to extract the demographic data (age, sex), operative time, surgical maneuvers performed, and surgical complications. Surgical records were evaluated to determine the specific maneuvers used on the nasal tip as well as other adjunctive procedures during the surgery. Patients with a history of injury, rhinoplasty or facial plastic surgery, who were followed-up for $<6$ months, or whose medical records were incomplete were excluded. This study was approved by the Institutional Review Board of Chungbuk National University Hospital (IRB No. 202003-006) and the need to obtain informed consent was waived.

\section{Surgical technique}

Each operation was performed by the senior author (WSS) and involved general anesthesia and an external approach. ECS was planned preoperatively when the patient had septal deviation and an esthetic need to improve his or her tip projection. Consent for publication of the patient images was obtained.

The operation procedure is described in Fig. 1. The osseocartilaginous skeleton was exposed with an external rhinoplasty approach, and the septal mucoperichondrial flaps were elevated. The entire septal cartilage was removed with the exception of the remaining about $10 \mathrm{~mm}$ of the dorsal stump in situ to avoid injury to the keystone. Twisted septal cartilage was then cut along

\section{H I G H L I G H T S}

- Increasing tip projection can be a challenge in rhinoplasty in Asian patients.

- Extracorporeal septoplasty achieves favorable esthetic and functional outcomes.

- This technique is useful for patients with a shortage of septal cartilage who desire tip refinement. the maximum deflection angle, and the neo- $\mathrm{L}$ strut was rebuilt with an optimal tip projection and dorsal profile using various techniques such as scoring, a batten graft, and a cutting and suture technique. A straighter and stronger caudal strut can be achieved using the posteroinferior portion of the septal cartilage, which is thicker than the anterior portion. The neo-L strut was then reimplanted into the anatomical position and fixed to the stump in the dorsum and to the soft tissues around the anterior nasal spine (ANS) in the middle using 4-0 polydioxanone sutures with a figure of eight feature. Additional spreader grafts were inserted to stabilize the dorsal support, if needed. After fixation of the neo-L strut, the dorsal part of the septal cartilage was reconnected to the upper lateral cartilage with 4-0 polydioxanone sutures. An additional tip onlay graft was added as necessary to improve tip definition. Finally, the skin incision was closed with 6-0 nylon sutures. External nasal splints were applied. The patients were followed-up at 1 week, 1 month, 3 months, 6 months, and 1 year after the surgery and on an annual basis thereafter. Two representative cases of ECS for rhinoplasty are presented in Figs. 2 and 3.

\section{Assessment of outcomes}

The surgical outcome was assessed with regard to subjective or objective evaluation and functional or esthetic improvement after $\geq 6$ months postoperatively. The degree of nasal obstruction symptoms was evaluated preoperatively and postoperatively using a visual analog scale (VAS) graded from 0 (no discomfort) to 10 (maximal symptoms). In addition, the subjective esthetic satisfaction of patients was classified as excellent, good, moderate, or poor using questionnaires completed at outpatient clinic visits.

Objective surgical outcomes were rated by two rhinoplasty surgeons who were not involved in the operations. The modified objective rhinoplasty outcome score (OROS) [10] was used to determine the objective surgical outcomes by comparing preoperative and postoperative standardized facial photographs. The postoperative result was graded using a four-point scale $(0$, poor outcome; 1 , no improvement; 2 , moderate outcome; 3 , good outcome; and 4, excellent outcome) evaluating eight factors: symmetry, dorsal height, dorsal length, dorsal width, tip width, tip rotation, tip projection, and overall results.

Anthropometric changes were assessed using preoperative and postoperative facial photographs taken $>6$ months postoperatively. The Image J ver. 1.46 (National Institutes of Health, Bethesda, MD, USA) was used for the measurement of the nasal findings. Tip projection change was assessed using lateral view facial photographs in order to assess the nasofrontal angle (NFA), nasolabial angle (NLA), and nasal tip projection index. The NFA was defined as the angle of the nasal tip, radix, and the most forward projecting point of the forehead. The NLA was defined as the angle between the line from the anterior columella to the subnasale and the line from the subnasale to the labrale superius. The tip projection index was measured using the Goode method 

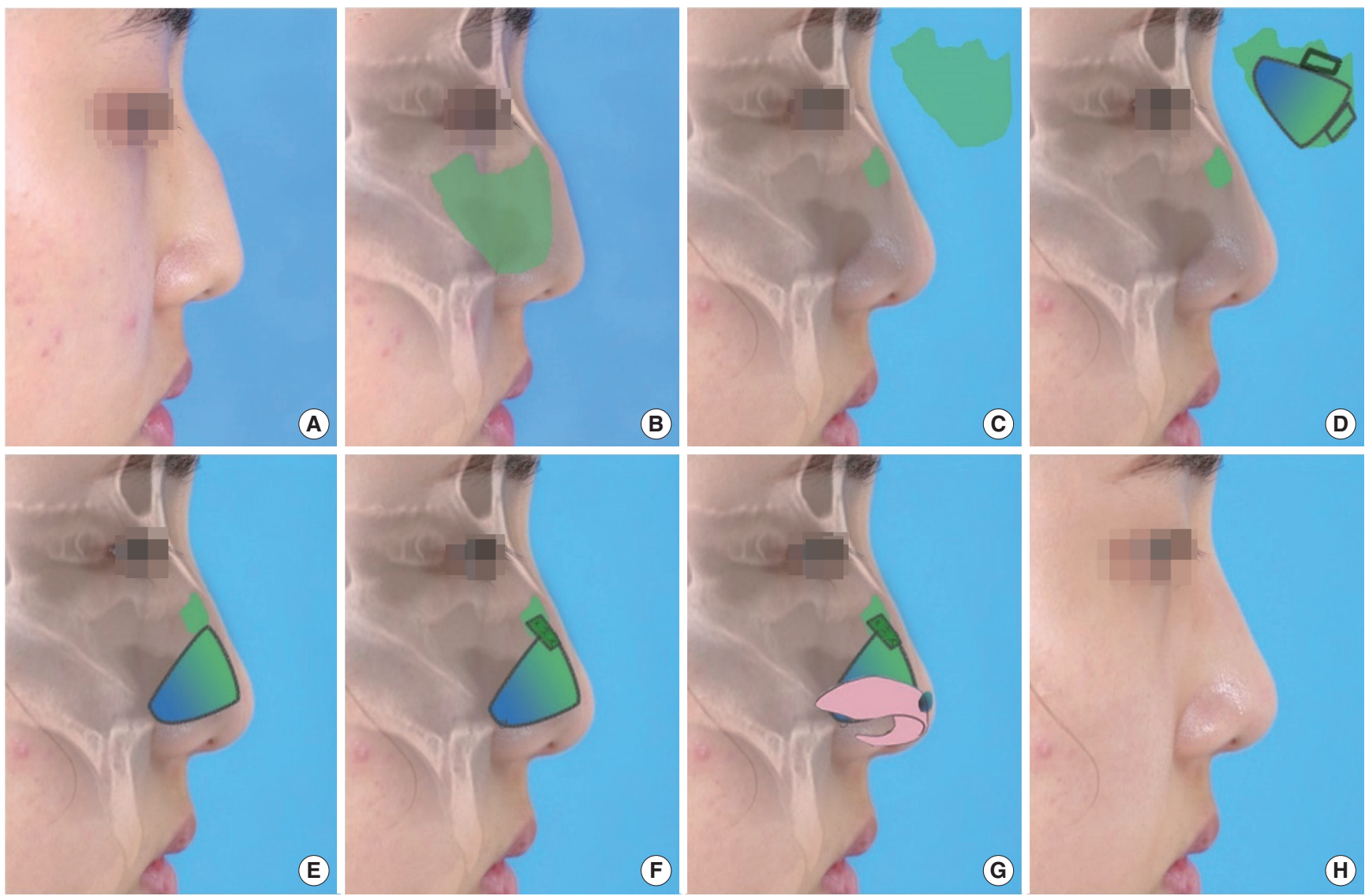

Fig. 1. Schematic drawing of the surgical procedure of rhinoplasty using the extracorporeal septoplasty technique. (A) Preoperative photograph of a patient illustrating tip drooping and a pseudohump. (B) Harvestable septal cartilage in situ. (C) Septal cartilage harvest with the exception of a few millimeters of the dorsal stump to avoid injury to the keystone area. (D) Extracorporeal design of the neo-L strut and spreader graft on the harvested septum. (E) Neo-L strut construction with an optimum tip projection and dorsal profile. (F) Additional spreader grafts for further stabilization of the dorsal support, if needed. (G) Additional onlay graft as necessary to improve tip definition. (H) Postoperative photograph of the patient with an improved dorsolateral profile.

(i.e., by drawing a line from the alar crease to the nasion, which is perpendicular to the line from the nasal tip to the alar crease; the length of the line drawn from the nasal tip to the alar crease is divided by the length of the nasion-to-nasal tip line). The degree of external nasal deviation was measured using the frontal view. Deviation angles were assessed between the vertical line between the midpoint of the glabella and the midpoint of the upper lip and another line that represented the nasal dorsal axis, consisting of the bony and cartilaginous parts from the nasion to the tip (Fig. 4) [11]. Other postoperative issues (loss of tip support or projection, recurrence of deviation, infection, and revision) were also evaluated.

\section{Statistical analysis}

The preoperative and postoperative degree of nasal obstruction and anthropometric measurements of the nose were compared using paired $t$-tests (SPSS ver. 12.0; SPSS Inc., Chicago, IL, USA). A $P$-value of $<0.05$ was considered to indicate statistical significance.

\section{RESULTS}

In total, 64 patients were included in this study, comprising 48 male and 16 female patients with a mean age of 29.3 years (range, $17-68$ years). All patients were followed-up for $\geq 10$ months, and the mean postoperative follow-up period was 13.3 months (range, 10-21 months). The mean operative time from incision to external splinting was 89.3 minutes. The concomitant procedures are listed in Table 1. For additional support of the neo-L strut or straightening of the dorsal septum, spreader grafts were required in 53 patients $(82.8 \%)$. Tip onlay grafting was performed in 44 patients $(68.8 \%)$ for additional tip definition. Additional conchal cartilage was needed in three patients.

\section{Surgical outcomes}

Endoscopic examination revealed a straight septum in all patients postoperatively, and the preoperative and postoperative VAS scores for nasal obstruction were $7.9 \pm 1.2$ and 3.1 \pm 1.3 , respectively $(P<0.001)$ (Fig. 5). Subjective patient satisfaction 

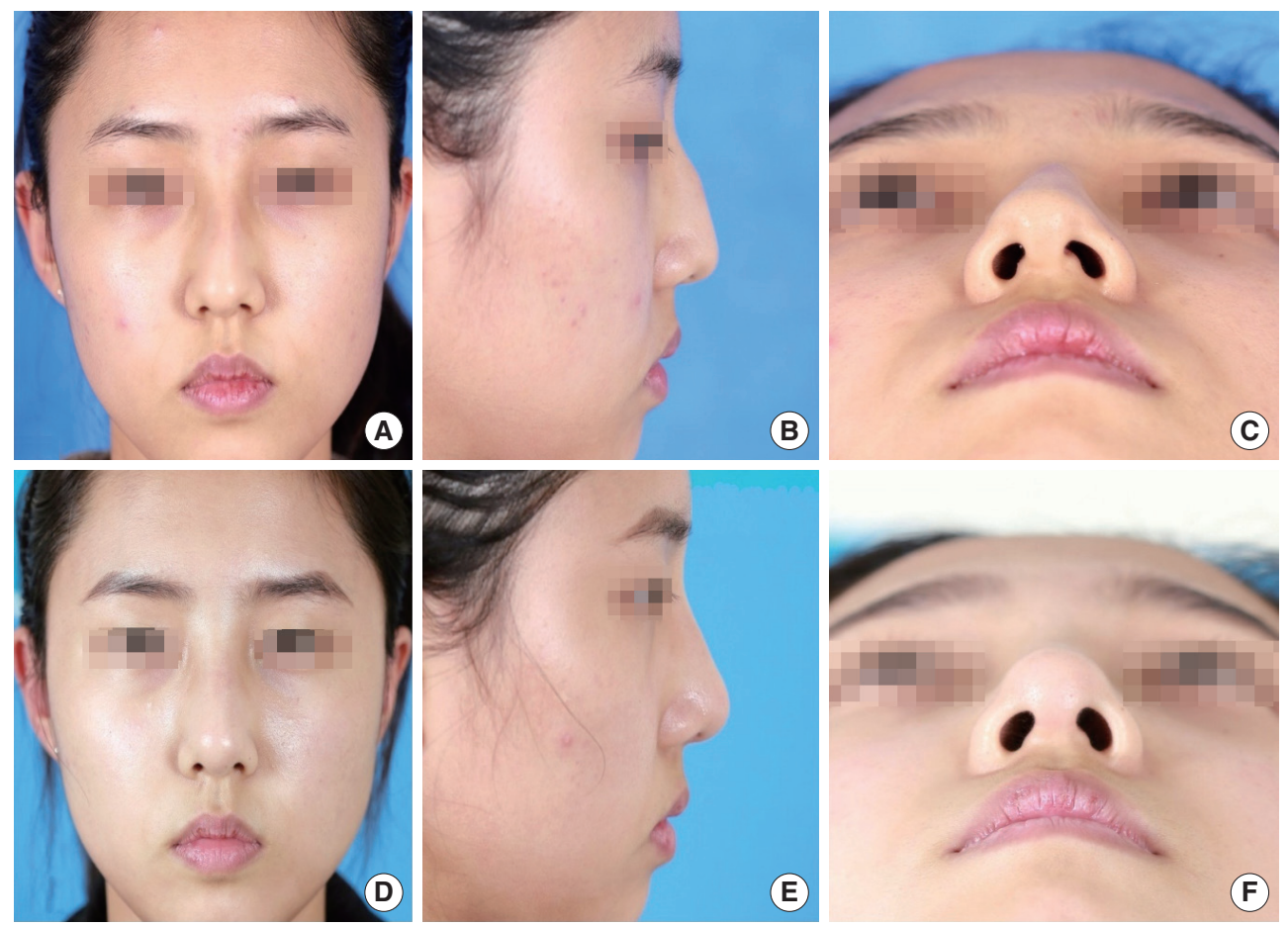

Fig. 2. Representative case. Preoperative (A-C) and 6-month postoperative (D-F) views of a 19-year-old woman with a deviated nose and underprojected tip treated with extracorporeal septoplasty for rhinoplasty (extracorporeal septoplasty technique with bilateral spreader grafts, two tip onlay grafts, and a dorsal onlay graft with crushed septal cartilage).
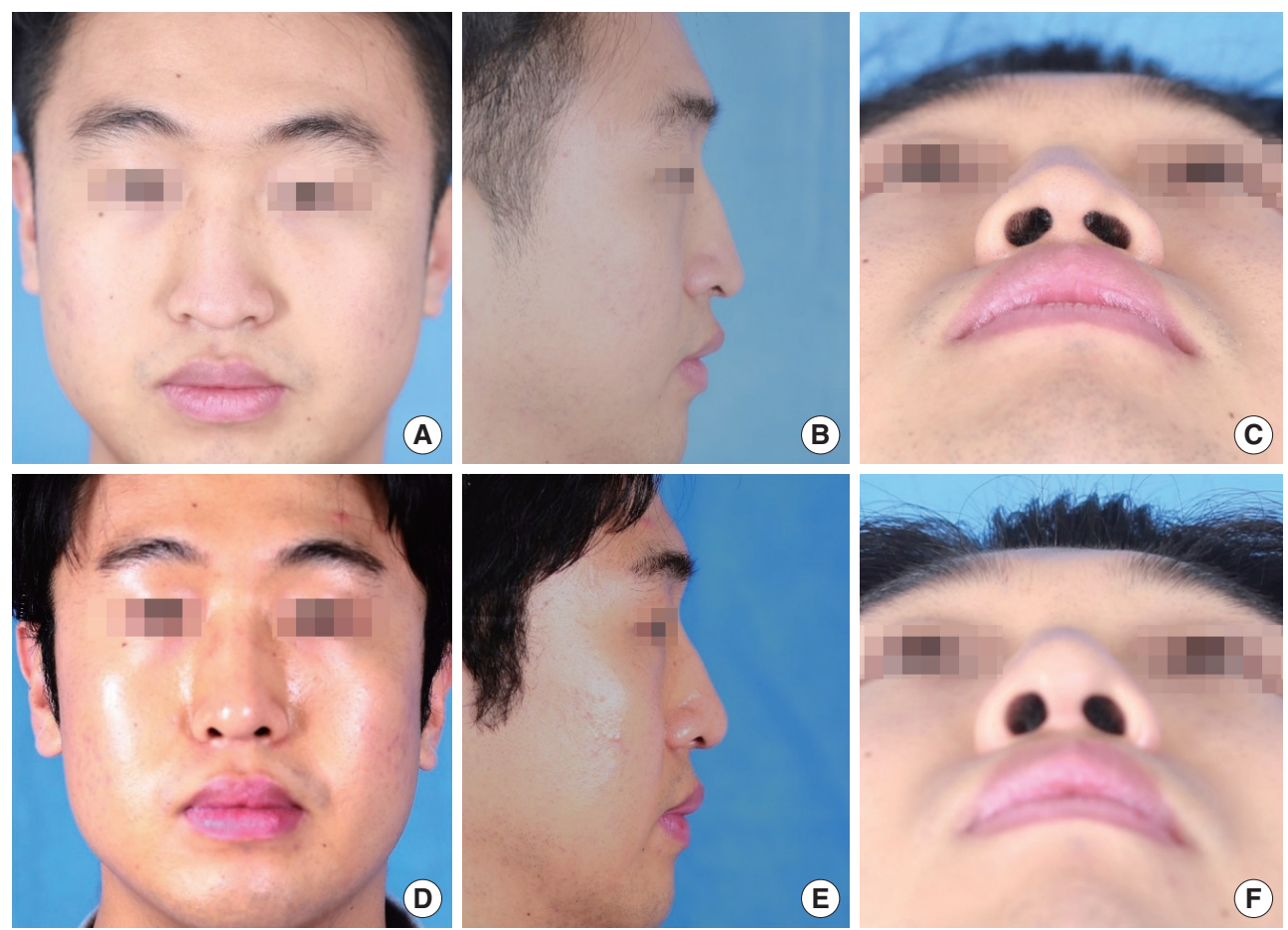

Fig. 3. Representative case. Preoperative (A-C) and 5-month postoperative (D-F) views of a 23-year-old man with an underprojected and derotated nasal tip treated with extracorporeal septoplasty for rhinoplasty (extracorporeal septoplasty technique with a unilateral spreader graft and a tip onlay graft). 

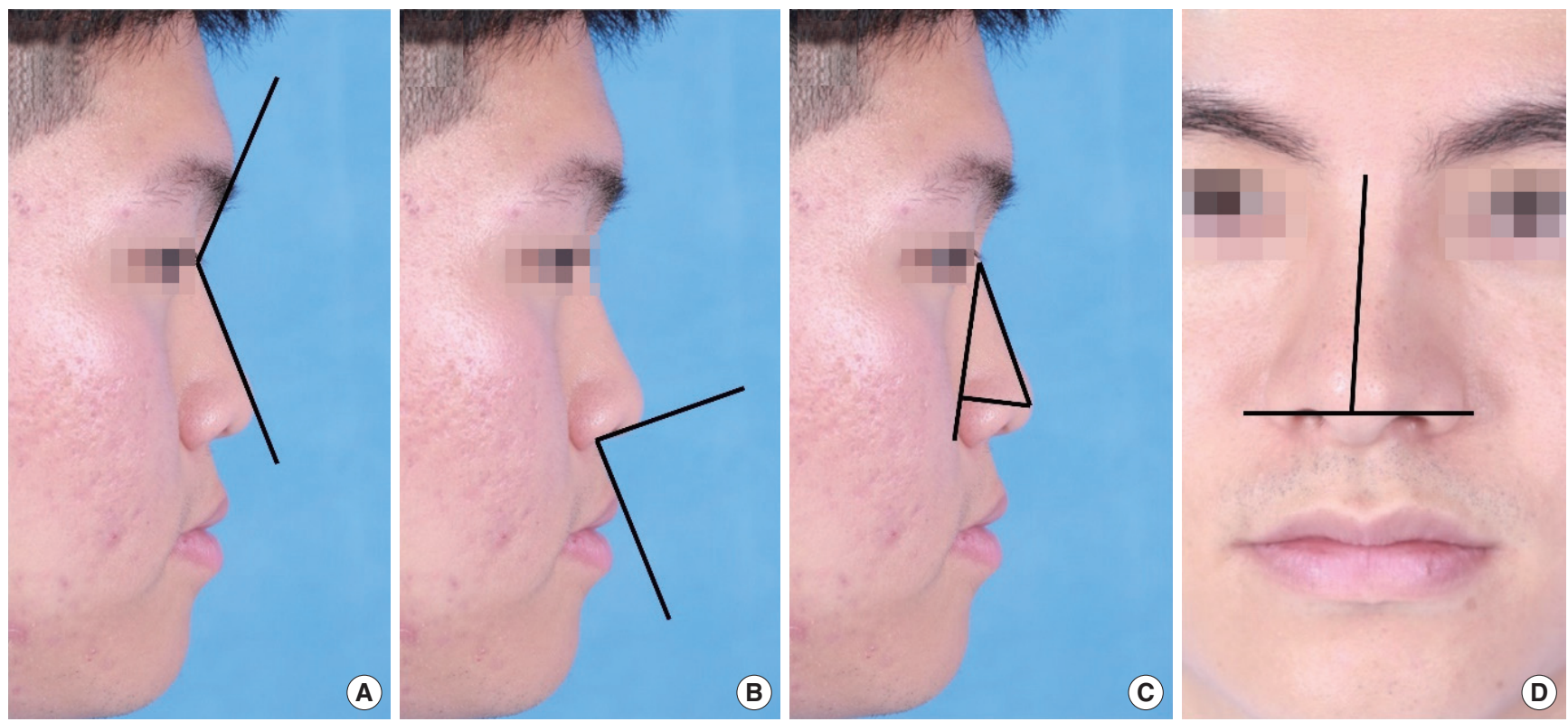

Fig. 4. Anthropometric measurements. (A) Nasofrontal angle: angle of the nasal tip, radix, and forward-most projecting point of the forehead. (B) Nasolabial angle: angle between the line from the anterior columella to the subnasale and the line from the subnasale to the labrale superius. (C) Tip projection index: the length of the line drawn from the nasal tip to the alar crease divided by the length of the nasion-to-nasal tip line. (D) Deviation angle: angle between the vertical line between the midpoint of the glabella and the midpoint of the upper lip and another line representing the nasal dorsal axis.

Table 1. Surgical procedures performed ${ }^{\text {a) }}$

\begin{tabular}{lc}
\hline Surgical procedure & No. of patients \\
\hline Spreader graft & 53 \\
Cap graft & 44 \\
Dorsal onlay graft & 38 (Silicone, 21; acellular dermal matrix, 17) \\
Osteotome & 11 \\
Septal extension graft & 0 \\
Conchal cartilage harvest & 3 \\
\hline
\end{tabular}

a)The numbers are not mutually exclusive.

regarding cosmetic results was excellent in 51 cases $(79.7 \%)$, good in 10 cases $(15.6 \%)$, moderate in three cases $(4.7 \%)$, and poor in 0 cases. The objective surgical outcomes as assessed using the OROS were $>3$ for all components, with an average overall result of 3.45 (Fig. 6).

There were significant changes in the postoperative NFA and NLA. The mean NFA was $152.3^{\circ} \pm 4.8^{\circ}$ preoperatively and $148.1^{\circ} \pm 3.7^{\circ}$ postoperatively $(P<0.001)$. The mean preoperative NLA was $88.8^{\circ} \pm 12.2^{\circ}$ and $92.0^{\circ} \pm 11.0^{\circ}$ postoperatively $(P<0.038)$. In addition, the tip projection index improved from $0.62 \pm 0.09$ preoperatively to $0.66 \pm 0.06$ postoperatively, which was a statistically significant change $(P<0.033)$. The postoperative change in the deviation angle was also statistically significant $\left(9.7^{\circ} \pm 7.6^{\circ}\right.$ preoperatively to $1.7^{\circ} \pm 3.2^{\circ}$ postoperatively, $P<0.001)$ (Table 2).

No major postoperative complications (e.g., infection, saddling, tip deviation, or reduction in tip projection) were noted

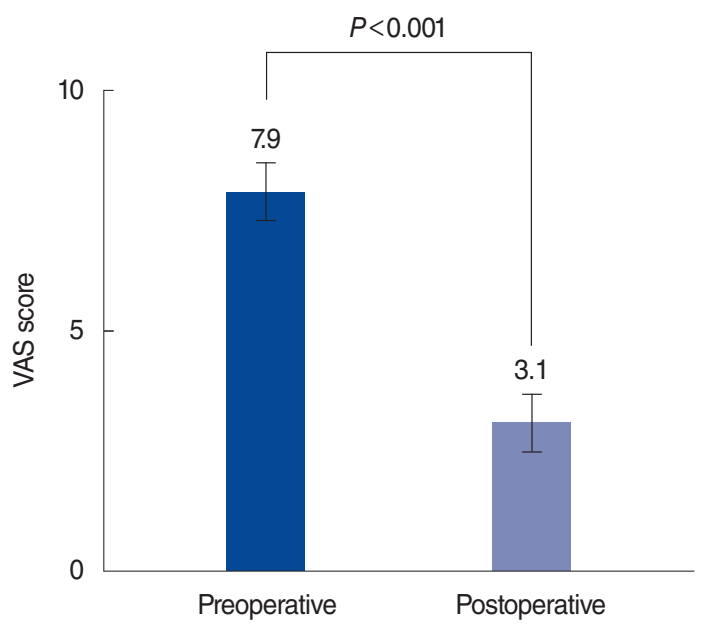

Fig. 5. Preoperative and postoperative visual analog scale (VAS) scores for nasal obstruction. The VAS scores for nasal obstruction significantly decreased from 7.9 to $3.1(P<0.001)$.

during the 13.3-month follow-up period. In addition, there were no cases of revision.

\section{DISCUSSION}

We evaluated the utility of the ECS technique in rhinoplasty in Asian patients with regard to functional and esthetic outcomes. Overall, esthetically satisfying outcomes were achieved, includ- 


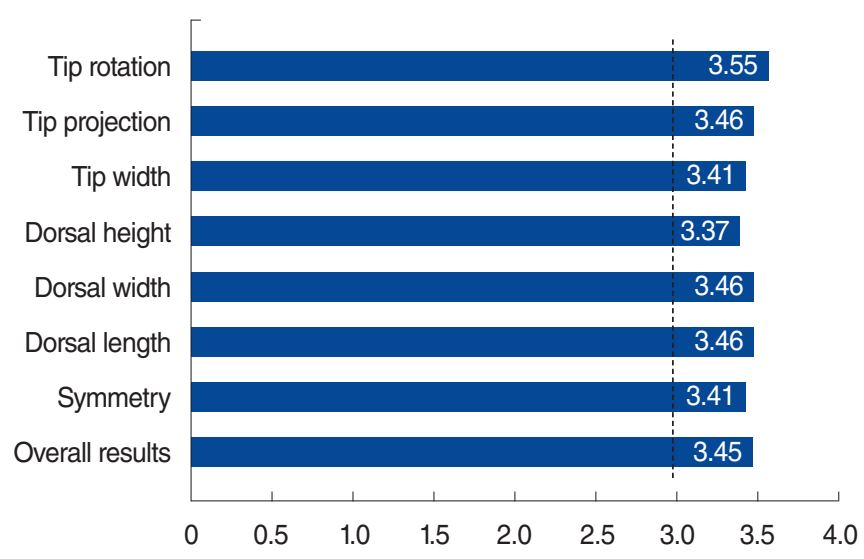

Fig. 6. Objective esthetic surgical outcomes using the objective rhinoplasty outcome score. The objective rhinoplasty outcome scores were $>3$ for all components, and the overall result was 3.45 .

ing tip projection, as well as significant improvements in nasal obstruction symptoms. Anthropometric measurements indicated that the ECS technique was successful in increasing tip projection and improving deviation correction.

The nasal tip of Asian patients is typically low, and Asian patients generally seek esthetic improvements such as greater projection and better definition of the tip [12]. Thus, tip surgery followed by dorsal augmentation is the most common rhinoplasty procedure for Asian patients [1]. Since the noses of Asian patients tend to have insufficient and weak septal cartilage and a thick soft-tissue and skin envelope, tip surgery can be challenging $[1,8,13]$. Many methods have been developed with the goal of overcoming these limitations and sufficiently improving nasal tip projection.

Since Byrd et al. [14] first reported the use of an SEG as an effective method to control the projection and rotation of the nasal tip, SEGs have been used frequently for rhinoplasty in Asian patients [8,14-16]. However, as the use of SEGs has increased, complications of this technique have also become more common [17-19]. The complications are related to insufficient or weak cartilage and thick skin, which are common in the noses of Asian patients [17-19]. Recurrent deviation has been reported in cases when an SEG is applied without fixation to the ANS [20], or in cases of a unilateral SEG fixed in a side-to-side manner with the tip position not exactly at the midline, resulting in pressure being exerted by the thick overlying skin and soft tissue envelope in the long term $[1,13,15,20]$. Additional batten grafts or extended spreader graft procedures may strengthen the caudal septum, but those procedures require much more cartilage, which is limited in Asian patients [13,15,20]. Additionally, adding a batten graft or extended spreader graft thickens the caudal septum, potentially causing nasal valve obstruction [15].

To overcome these limitations of SEGs, ECS can be applied as an attractive alternative technique for rhinoplasty in Asian patients. In particular, ECS is a useful septoplasty technique for
Table 2. Preoperative and postoperative anthropometric measurements

\begin{tabular}{lccr}
\hline Variable & Preoperative & Postoperative & $P$-value \\
\hline Nasofrontal angle & $152.3 \pm 4.8$ & $148.1 \pm 3.7$ & $<0.001$ \\
Nasolabial angle & $88.8 \pm 12.2$ & $92.0 \pm 11.0$ & 0.038 \\
Tip projection index & $0.62 \pm 0.09$ & $0.66 \pm 0.06$ & 0.033 \\
External deviation angle & $9.7 \pm 7.6$ & $1.7 \pm 3.2$ & $<0.001$ \\
\hline
\end{tabular}

Values are presented as mean \pm standard deviation.

the surgical treatment of severely deviated septa. In ECS, the entire septum is removed, reshaped, and then reimplanted back into its original site [1,9]. As the amount of harvested cartilage is greater than that in classic septoplasty, if designed properly, all of the small-to-medium sized working pieces of harvested cartilage can be effectively used as the neo-L strut and other grafts, if needed. Harvesting the maximal possible amount of cartilage may enable secure fixation of the neo-L strut onto the ANS and reinforcement of the keystone area with a spreader graft. In the technique that we have proposed, the thicker inferoposterior portion of the original septal cartilage is rotated caudally as the caudal part of the neo-L strut. In this manner, strong support of the caudal septum becomes available, and cartilage is not wasted on additional reinforcement or structuring, such as a columellar strut. In the present study, autologous conchal cartilage was used in $4.7 \%$ (3/64) of cases to provide additional cartilage support. If the ECS is connected to the dorsal strip in an end-toend manner, the likelihood of recurrent deviation in the long term seems to be reduced, as the septum is fixed at the midline. However, long-term follow-up is needed to confirm this theory, as the mean follow-up time in the current study was relatively short. In addition, the strong neo-L strut of the ECS technique minimized the need for additional septal batten grafts, thereby reducing caudal septum thickening in comparison to SEGs.

Additionally, the ECS technique is useful for achieving cosmetic improvements, as the surgeon can control the tip position of the neo-L strut and thereby increase tip projection and rotation. A previous report mentioned that this esthetic role of ECS obviates the need for additional tip grafting because the height of the tip can easily be changed by adjusting the caudal height of the extracorporeally-designed septal cartilage neo-L strut [1]. ECS has been reported to be a useful technique for markedly deviated noses, as it can achieve comparable esthetic success and better functional outcomes than conventional septoplasty in rhinoplasty [21]. Our study confirmed these results via an anthropometric analysis, as the ECS technique yielded significant improvements in tip projection and external nose deviation correction. The deviated septum was straightened in each case, as confirmed by an endoscopic exam. The VAS score for nasal obstruction symptoms was reduced in all cases. The lack of caudal septum thickening with the ECS technique may have contributed to subjective symptom improvements. Postoperative patient satisfaction was rated as "excellent" or "good" by $95.3 \%$ of the 
patients, and there were no dissatisfied patients with "poor" results. The objective esthetic results were $>3$ of 5 for every component of the OROS.

Although ECS has been advocated as an efficient procedure for tip augmentation in Asian patients, a major drawback is its technical difficulty, because the surgeon is required to separate the original septal cartilage en bloc from the ANS and keystone areas. Therefore, inexperienced rhinoplasty surgeons may hesitate to perform ECS. Achieving secure fixation at the ANS and keystone areas remains a technical challenge for even experienced rhinoplasty surgeons. However, the authors believe that surgeons can overcome this fear and hesitation by simplifying the procedure of rhinoplasty with ECS. Septoplasty in rhinoplasty is mainly focused on achieving three goals: first, to obtain a straight septum dorsally and caudally, alleviating its contribution to deviation; second, to restore adequate septal support that is capable of withstanding added dorsal onlay grafts and the overlying pressure of the skin and the soft tissue envelope; and third, to obtain graft material for rhinoplasty $[22,23]$. ECS can be used to accomplish all three of those goals. By designing the neo-L strut extracorporeally, the need for an extra graft, such as a batten graft, to support the septum is reduced, making preoperative planning easier and the results more predictable. In the authors' experience, performing consistent and repeated procedures also contributed to reduced operative time.

Our indications for ESC have continued to broaden as we have early cases to include a wide spectrum of cases. In addition, our indications for ECS have continued to broaden as we have gained more technical experience. In contrast to a previous report, the mean operative time was 90 minutes, which is similar to or shorter than that of conventional rhinoplasty [21]; this occurred because the procedures were simplified, and additional cartilage harvesting was not needed in the majority of cases. A previous report described the possibility of septal perforation due to submucosal bilateral tunneling in ECS [24]. However, there were no such complications in our study.

The present study has some limitations, principally stemming from its retrospective design. The study involved a relatively small number of patients, a relatively short follow-up period, and no surgical control group. An additional limitation of this study that by additionally performing a tip onlay graft in about $70 \%$ of cases, the authors were not able to assess the sole effect of the modified ECS technique on tip projection. However, in all cases, the authors observed that the tip projection was significantly improved by using the modified ECS technique, and the tip onlay graft was only performed an add-on procedure to achieve greater tip definition.

In conclusion, the ECS technique in rhinoplasty in Asian patients achieved effective and reliable results in terms of esthetic and functional outcomes. The shortage of septal cartilage and caudal support in the noses of Asian patients was overcome with the ECS technique by harvesting a sufficient amount of cartilage. Functional outcomes were improved by reducing caudal septal thickening. It is expected that the ECS technique may play an broader role in rhinoplasty in Asian patients.

\section{CONFLICT OF INTEREST}

No potential conflict of interest relevant to this article was reported.

\section{ORCID}

Sue Jean Mun https://orcid.org/0000-0003-4486-7178

Young-Seok Choi https://orcid.org/0000-0001-5139-9830

Ji Hwan Kim https://orcid.org/0000-0002-0168-9183

Woo Sub Shim https://orcid.org/0000-0003-2236-0368

Hahn Jin Jung ～https://orcid.org/0000-0002-6015-7048

\section{AUTHOR CONTRIBUTIONS}

Conceptualization: WSS, HJJ. Data curation: YSC, SJM, JHK. Formal analysis: SJM. Methodology: YSC, WSS, HJJ. Investigation: JHK. Writing-original draft: HJJ, SJM, JHK. Writing-review \& editing: YSC, WSS, HJJ.

\section{REFERENCES}

1. Jang YJ,Alfanta EM. Rhinoplasty in the Asian nose. Facial Plast Surg Clin North Am. 2014 Aug;22(3):357-77.

2. Jang YJ. Dorsal augmentation using costal cartilage: what is the best way? Clin Exp Otorhinolaryngol. 2019 Nov;12(4):327-8.

3. Matory WE Jr, Falces E. Non-Caucasian rhinoplasty: a 16-year experience. Plast Reconstr Surg. 1986 Feb;77(2):239-52.

4. Aung SC, Foo CL, Lee ST. Three dimensional laser scan assessment of the Oriental nose with a new classification of Oriental nasal types. Br J Plast Surg. 2000;53(2):109-16.

5. WuWT.The Oriental nose: an anatomical basis for surgery. Ann Acad Med Singapore. 1992 Mar;21(2):176-89.

6. Zingaro EA, Falces E.Aesthetic anatomy of the non-Caucasian nose. Clin Plast Surg. 1987 Oct;14(4):749-65.

7. Han SK, Lee DG, Kim JB, Kim WK. An anatomic study of nasal tip supporting structures. Ann Plast Surg. 2004 Feb;52(2):134-9.

8. Choi JY, Kang IG, Javidnia H, Sykes JM. Complications of septal extension grafts in Asian patients. JAMA Facial Plast Surg. 2014 MayJun;16(3):169-75.

9. Jang YJ, Kwon M. Modified extracorporeal septoplasty technique in rhinoplasty for severely deviated noses. Ann Otol Rhinol Laryngol. 2010 May;119(5):331-5.

10. Park P, Jin HR. Diced cartilage in fascia for major nasal dorsal augmentation in Asians: a review of 15 consecutive cases. Aesthetic Plast Surg. 2016 Dec;40(6):832-9.

11. Choi JY, Park JH, Javidnia H, Sykes JM. Effect of various facial angles and measurements on the ideal position of the nasal tip in the Asian patient population. JAMA Facial Plast Surg. 2013 Nov-Dec;15(6): 
417-21.

12. Wang JH, Jang YJ, Park SK, Lee BJ. Measurement of aesthetic proportions in the profile view of Koreans. Ann Plast Surg. 2009 Feb; 62(2):109-13.

13. Kim JS, Khan NA, Song HM, Jang YJ. Intraoperative measurements of harvestable septal cartilage in rhinoplasty. Ann Plast Surg. 2010 Dec;65(6):519-23.

14. Byrd HS, Andochick S, Copit S, Walton KG. Septal extension grafts: a method of controlling tip projection shape. Plast Reconstr Surg. 1997 Sep;100(4):999-1010.

15. Jin HR, Won TB. Nasal tip augmentation in Asians using autogenous cartilage. Otolaryngol Head Neck Surg. 2009 Apr;140(4):526-30.

16. Ha RY, Byrd HS. Septal extension grafts revisited: 6-year experience in controlling nasal tip projection and shape. Plast Reconstr Surg. 2003 Dec;112(7):1929-35.

17. Kang JG, Ryu J. Nasal tip surgery using a modified septal extension graft by means of extended marginal incision. Plast Reconstr Surg. 2009 Jan;123(1):343-52.

18. Emsen IM.A different approach to the reconstruction of the stubborn crooked nose with a different spreader graft: nasal bone grafts har- vested from them removed nasal hump. Aesthetic Plast Surg. 2008 Mar;32(2):266-73.

19. Gunter JP, Rohrich RJ. Lengthening the aesthetically short nose. Plast Reconstr Surg. 1989 May;83(5):793-800.

20. Lin J, Chen X, Wang X, Gao X, Zheng X, Chen X, et al.A modified septal extension graft for the Asian nasal tip. JAMA Facial Plast Surg. 2013 Sep-Oct;15(5):362-8.

21. Lee SB, Jang YJ. Treatment outcomes of extracorporeal septoplasty compared with in situ septal correction in rhinoplasty. JAMA Facial Plast Surg. 2014;16(5):328-34.

22. Won TB, Kang JG, Jin HR. Management of post-traumatic combined deviated and saddle nose deformity. Acta Otolaryngol. 2012 Jun;132 Suppl 1:S44-51.

23. Jin HR, Kim DW, Jung HJ. Common sites, etiology, and solutions of persistent septal deviation in revision septoplasty. Clin Exp Otorhinolaryngol. 2018 Dec;11(4):288-92.

24. Kantas I, Balatsouras DG, Papadakis CE, Marangos N, Korres SG, Danielides V. Aesthetic reconstruction of a crooked nose via extracorporeal septoplasty. J Otolaryngol Head Neck Surg. 2008 Apr; 37(2):154-9. 